\title{
Retraction
}

\section{Retracted: Is Hippocampus Susceptible to Antinociceptive Tolerance to NSAIDs Like the Periaqueductal Grey?}

\author{
Pain Research and Treatment \\ Received 12 October 2015; Accepted 12 October 2015 \\ Copyright (C) 2015 Pain Research and Treatment. This is an open access article distributed under the Creative Commons Attribution \\ License, which permits unrestricted use, distribution, and reproduction in any medium, provided the original work is properly cited.
}

The paper titled "Is Hippocampus Susceptible to Antinociceptive Tolerance to NSAIDs Like the Periaqueductal Grey?" [1] has been retracted as it is essentially identical in content with a previously published paper titled "Antinociceptive Tolerance to NSAIDs Microinjected into Dorsal Hippocampus" published by the same authors in BMC Pharmacology and Toxicology.

\section{References}

[1] N. Tsiklauri, I. Nozadze, G. Gurtskaia, and M. G. Tsagareli, "Is hippocampus susceptible to antinociceptive tolerance to NSAIDs like the periaqueductal grey?" Pain Research and Treatment, vol. 2014, Article ID 654578, 8 pages, 2014. 


\title{
Is Hippocampus Susceptible to Antinociceptive Tolerance to NSAIDs Like the Periaqueductal Grey?
}

\author{
Nana Tsiklauri, Ivliane Nozadze, Gulnazi Gurtskaia, and Merab G. Tsagareli \\ Department of Neurophysiology, Ivane Beritashvili Center for Experimental Biomedicine, 0160 Tbilisi, Georgia \\ Correspondence should be addressed to Merab G. Tsagareli; tsagareli@biphysiol.ge
}

Received 3 February 2014; Revised 6 March 2014; Accepted 21 March 2014; Published 9 April 2014

Academic Editor: Donald A. Simone

Copyright (C) 2014 Nana Tsiklauri et al. This is an open access article distributed under the Creative Commons Attribution License, which permits unrestricted use, distribution, and reproduction in any medium, provided the original work is properly cited.

\begin{abstract}
Emotional distress is the most undesirable feature of painful experience. Numerous studies have demonstrated the important role of the limbic system in the affective-motivational component of pain. The purpose of this paper was to examine whether microinjection of nonsteroidal anti-inflammatory drugs (NSAIDs), Clodifen, Ketorolac, and Xefocam, into the dorsal hippocampus (DH) leads to the development of antinociceptive tolerance in male rats. We found that microinjection of these NSAIDs into the $\mathrm{DH}$ induces antinociception as revealed by a latency increase in the tail-flick (TF) and hot plate (HP) tests compared to controls treated with saline into the $\mathrm{DH}$. Subsequent tests on consecutive three days, however, showed that the antinociceptive effect of NSAIDs progressively decreased, suggesting tolerance developed to this effect of NSAIDs. Both pretreatment and posttreatment with the opioid antagonist naloxone into the DH significantly reduced the antinociceptive effect of NSAIDs in both pain models. Our data indicate that microinjection of NSAIDs into the $\mathrm{DH}$ induces antinociception which is mediated via the opioid system and exhibits tolerance.
\end{abstract}

\section{Introduction}

Emotional distress is an intrinsic and the most disruptive and undesirable feature of painful states. Pain is characterized as a complex experience, dependent not only on the regulation of nociceptive sensory systems but also on the activation of mechanisms that control emotional processes in limbic brain areas such as the amygdala and the hippocampus $[1,2]$. First experiments in Melzack's laboratory by injection of local anesthetics into limbic structures show a temporary block of neural activity and an induction of significant analgesia during late tonic pain perception [35]. The involvement of the hippocampal formation (HF) in nociception has been suggested in several studies [5-7]. Some abnormalities in hippocampal functioning with persistent pain have been recently shown [8]. Particularly, mice with spared nerve injury (SNI) neuropathic pain were unable to extinguish contextual fear and showed increased anxiety-like behavior. Additionally, mice with SNI compared with sham animals exhibited hippocampal reduced extracellular signalregulated kinase expression and phosphorylation, decreased neurogenesis, and altered short-term synaptic plasticity [8].
Furthermore, morphine microinjections in the dorsal hippocampus $(\mathrm{DH})$ produced antinociceptive effects in the formalin-induced orofacial pain model in rats [9]. Recent evidence suggests the participation of cholinergic, opioidergic, and GABAergic systems of the DH in the modulation of nociception in guinea pigs [6]. Moreover, opioid peptides are important modulators of information processing in the hippocampus. When activated, opioid receptors play a key role in central pain modulation mechanisms, and the HF is a structure that expresses significant densities of this kind of receptors $[10,11]$. In addition, the hippocampus is anatomically connected to components of the pain neuromatrix, including the amygdala and the descending pain pathway with the periaqueductal gray (PAG) - the rostral ventromedial (RVM) region of medulla [12-14]. However, specific neural substrates and circuitry mediating opioidinduced hippocampal antinociception are unknown.

We have recently shown that, in the PAG, the central nucleus of amygdala (CeA), and the nucleus raphe magnus (NRM), microinjection of nonsteroidal anti-inflammatory drugs (NSAIDs) induces antinociception with some effects of tolerance and cross-tolerance to morphine [15-19]. These 
findings strongly support the suggestion of endogenous opioid involvement in NSAIDs antinociception and tolerance in the descending pain-control system [20-24]. However, involvement of NSAIDs antinociception in the HF is still a matter of controversy. For example, indomethacin did not protect against significant pain-induced downregulation of neurokinin-1 (NK-1) and brain-derived neurotrophic factor (BDNF) receptor genes in the hippocampus, suggesting that although analgesic drug treatment reduces nociceptive sensory activation in the spinal cord, it is insufficient to prevent the impact of pain on the hippocampus [25].

In this study, we have examined whether microinjection of the widely used NSAIDs, Clodifen, Ketorolac, and Xefocam, into the DH induces antinociceptive tolerance and whether this action is mediated via the endogenous opioid system.

\section{Materials and Methods}

2.1. Animals. The experiments were carried out on male Wistar rats, 200-250 g in body weight, bred at Beritashvili Center for Experimental Biomedicine. The experimental protocol was approved by the local bioethical committee of the center. Every effort was made to minimize both the number of animals used and their suffering. Guidelines of the International Association for the Study of Pain regarding animal experimentation were followed throughout [26].

2.2. Surgical Procedures. Under anesthesia with thiopental (55 mg/kg, i.p., "Kievmed," Ukraine), a 25-gauge stainless steel guide cannula (Small Parts, Inc., USA) was stereotaxically implanted into the DH bilaterally (AP: $-4.3 ; \mathrm{L}: \pm 2.5$; $\mathrm{H}$ : 2.8 ) according to the coordinates in the atlas of Paxinos and Watson [27] siting the tip $2 \mathrm{~mm}$ above the DH. Guides were anchored to the cranium by dental cement. The guide cannula was plugged with a stainless steel stylet. Thereafter, the rats were handled every day for 3 days for $15 \mathrm{~min}$. During this time, the stylet was removed and a 30-gauge stainless steel microinjection cannula was inserted into the guide cannula to reach the $\mathrm{DH}$, but no drug was injected. This helped to habituate the rats to the injection procedure and to reduce artifacts arising from mechanical manipulation during the test days. Five days after surgery, a microinjection cannula, attached to a Hamilton syringe, was introduced through the guide cannula, and the drug was microinjected while the rat was gently restrained.

2.3. Drugs. Clodifen (diclofenac sodium, $75 \mu \mathrm{g} / 0.5 \mu \mathrm{L}$, RotexMedica, Germany), Ketorolac (ketorolac tromethamine, $90 \mu \mathrm{g} / 0.5 \mu \mathrm{L}$, Zee Drugs, India), or Xefocam (lornoxicam, $12 \mu \mathrm{g} / 0.5 \mu \mathrm{L}$, Nycomed, Austria) was injected through the microinjection cannula; the guide cannula was then plugged with a stainless steel stylet. Saline was injected in the same volume $(0.5 \mu \mathrm{L}$, GalichPharm, Ukraine) and manner in a separate group of rats for controls. Solutions were microinjected in about 10 seconds.
2.4. Behavioral Testing. Twenty minutes after microinjection, that is, $10 \mathrm{~min}$ before the peak of the drugs' effect is normally reached, animals were tested for antinociception using the tail-flick (TF) and hot plate (HP) tests. For the TF test, the distal part of the tail was stimulated with a light beam and the latency measured until the tail was reflexively flicked away from the beam (IITC \#33, IITC Life Science, Inc., Woodland Hills, CA, USA). For the HP test, the rat was placed on a $55^{\circ} \mathrm{C}$ hot plate and the latency to the first hindpaw licking or jumping was measured (IITC \#39). The cut-off time was $20 \mathrm{~s}$ for both TF and HP latencies. Each rat was tested with both tail-flick (TF) and hot plate (HP) in the same session. A similar procedure was followed for the repeated microinjection of Clodifen, Ketorolac, Xefocam, or saline for four consecutive days.

In control experiments, saline microinjections into the $\mathrm{DH}$ were followed by a nonselective opioid receptor antagonist naloxone ( $0.5 \mu \mathrm{L}$, GalichPharm, Ukraine) and tested for TF and HP latencies. Naloxone was treated 20 minutes after saline microinjections.

In the second set of experiments, twenty minutes after NSAIDs administration, we tested whether posttreatment with a nonselective opioid receptor antagonist naloxone in the DH diminishes NSAID-induced antinociception.

In the third set of experiments, rats were pretreated with the same dose of naloxone in the DH and tested with TF and HP latencies. $10 \mathrm{~min}$ after, rats were treated with NSAIDs in the same dose as in the first and second sets of experiments and were then tested again. Different animal groups were used for experiments 1,2 , and 3 . The number of rats in each group was five or six.

2.5. Histology. At the end of each set of experiments, the microinjection sites were marked with $2 \mu \mathrm{L}$ of saturated solution of Pontamine Sky Blue (Sigma Chemical, Co.) and the animal was euthanized with an overdose of diethyl ether. After fixation by immersion in $10 \%$ formalin, the brain was sectioned and counterstained with Cresyl Violet. The microinjection sites were histologically verified and plotted according to Paxinos and Watson (1997) stereotaxic atlas coordinates [27]. Representative microinjection sites are shown in Figure 1.

2.6. Statistical Analysis. All data are presented as mean \pm S.E.M. Repeated measures of analysis of variance (ANOVA) with post hoc Tukey-Kramer multiple comparison test were used for statistical comparisons between treated and saline groups and treated and naloxone groups, respectively. The Kolmogorov-Smirnov test was applied to verify normality. The statistical software utilized was InStat 3.05 (GraphPad Software, USA). Statistical significance between vehicle control and treated groups and naloxone and treated groups of rats was acknowledged if $P<0.05$.

\section{Results}

We found that microinjection of NSAIDs into the DH produced antinociception as revealed by a latency increase 


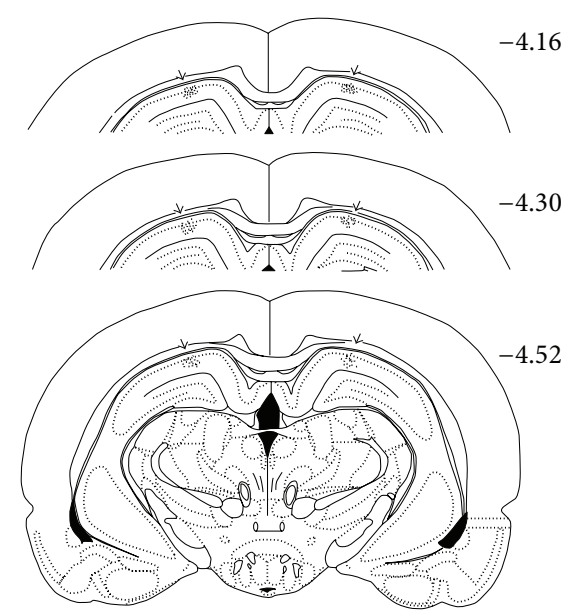

FIGURE 1: Serial coronal sections of the rat brain showing placement of microinjections bilaterally in the $\mathrm{DH}$ (black arrows). The numbers to the right of the sections represent millimeters relative to bregma, adapted from the Paxinos and Watson (1997) stereotaxic atlas [27].

in TF and HP compared to the baseline control of intact rats and a control group with saline microinjected into the same site as well. The TF latency significantly increased for Clodifen (ANOVA: $F(4,16)=20.189, P<0.0001$ ), Ketorolac (ANOVA: $F(4,20)=22.314, P<0.0001$ ), and Xefocam (ANOVA: $F(4,16)=32.42, P<0.0001$ ). We found similar significant differences in the HP latencies for Clodifen (ANOVA: $F(4,16)=21.53, P<0.0001$ ), for Ketorolac (ANOVA: $F(4,20)=17.764, P<0.0001$ ), and for Xefocam (ANOVA: $F(4,16)=39.463, P<0.0001$ ), respectively. Subsequent NSAIDs microinjections caused progressively less antinociception, so by day 4 there was no effect, similar to saline microinjections for both the TF and the HP tests (see Figure 2).

Control testing with saline microinjections into the $\mathrm{DH}$ followed by a nonselective opioid receptor antagonist naloxone statistically did not change the latency to respond in the TF (ANOVA: $F(5,24)=0.8914, P=0.5024$, not significant) and HP (ANOVA: $F(5,24)=0.1463, P=0.9792$, not significant) tests, respectively $(P>0.05)$ (see Figures $3(\mathrm{a})$ and 3(b)).

In the second set of experiments, we tested if posttreatment with the nonselective opioid receptor antagonist naloxone in the DH diminishes NSAID-induced antinociception. Twenty minutes after NSAID administration, microinjection of naloxone in the DH significantly decreased antinociceptive effects of these drugs in the TF for Clodifen (ANOVA: $F(5,20)=26.906, P<0.0001)(t=13.161, P<0.001)$ (Figure 4(a)), for Ketorolac (ANOVA: $F(5,20)=24.701$, $P<0.0001)(t=10.691, P<0.001)$ (Figure 4(b)), and for Xefocam (ANOVA: $F(5,20)=22.412, P<0.0001$ ) $(t=9.745, P<0.001)$, respectively (Figure $4(\mathrm{c}))$.

We discovered the same effects in the HP test for Clodifen (ANOVA: $F(5,20)=11.341, P<0.0001)(t=6.679, P<$ $0.01)$ (see Figure 5(a)), for Ketorolac (ANOVA: $F(5,20)=$ 33.093, $P<0.0001)(t=12.141, P<0.001)$ (see Figure 5(b)),
TABLE 1: Values (mean \pm S.E.M.) for saline, naloxone, and NSAIDs groups for TF and HP tests.

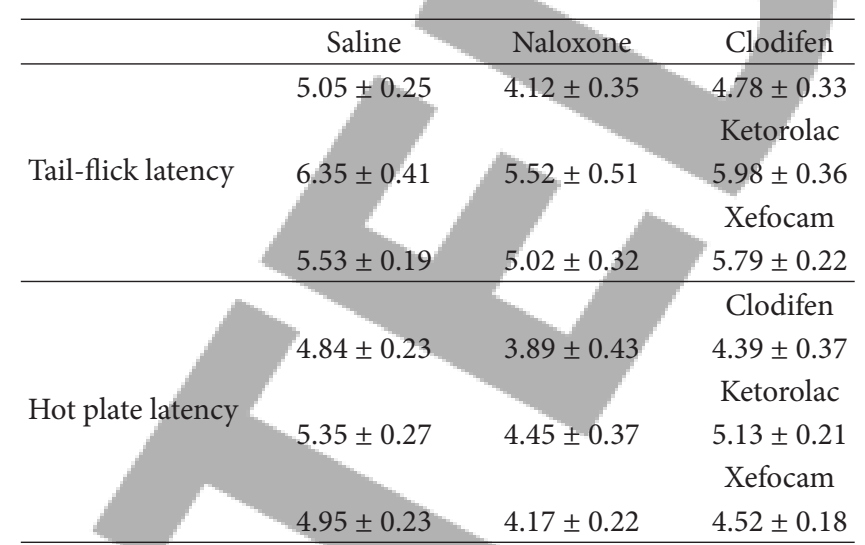

Comparisons between groups do not show significance $(P>0.05)$.

ANOVA with post hoc test. Number of rats: $N=5$ /groups.

and for Xefocam (ANOVA: $F(5,20)=35.494, P<0.0001$ )

$(t=13.068, P<0.001)$, respectively (see Figure 5(c)).

In the third set of experiments, we tested if pretreatment with naloxone prevents antinociception induced by NSAID microinjected into the DH. Pretreatment with naloxone completely prevented the analgesic effects of Clodifen, Ketorolac, and Xefocam in the TF test. The differences between NSAIDs injected and naloxone injected groups are not significant $(P>0.05)$. The same results are in the HP test for Clodifen, Ketorolac, and Xefocam, respectively (see Table 1).

\section{Discussion}

The present results demonstrate that microinjections of the NSAIDs, Clodifen, Ketorolac, and Xefocam, into the $\mathrm{DH}$ induce antinociception. This confirms our and other colleagues previous results with systemic, intraperitoneal administration of NSAIDs $[28,29]$ and results using microinjection of the same NSAIDs into the PAG [20,21]. In the other experiments in rats, responses of spinal dorsal horn widedynamic range neurons to mechanical noxious stimulation of a hindpaw were strongly inhibited by intravenous NSAID dipyrone (metamizole) [30]. Importantly, repeated microinjections of NSAIDs into the DH resulted in a progressive decrease in antinociceptive effectiveness, that is, induced tolerance similar to that observed with intra-PAG, CeA, and NRM injections [16, 17, 19-22], and reminiscent of the effect of opiates.

A major involvement of opioidergic mechanisms in tolerance to the analgesic effect of NSAIDs is unusual, because the cellular and molecular actions of opioids were thought to differ from those of nonopioid analgesics. However, one interesting aspect of NSAIDs administration, namely, tolerance, emphasizes their similarity to opioid analgesics. Indeed, microinjection of dipyrone (metamizole) into PAG [18, 20, $21,23]$ or into CeA $[15,16,23]$ progressively led to a loss of their antinociceptive effects, that is, produced tolerance. Furthermore, tolerance to dipyrone was accompanied by 


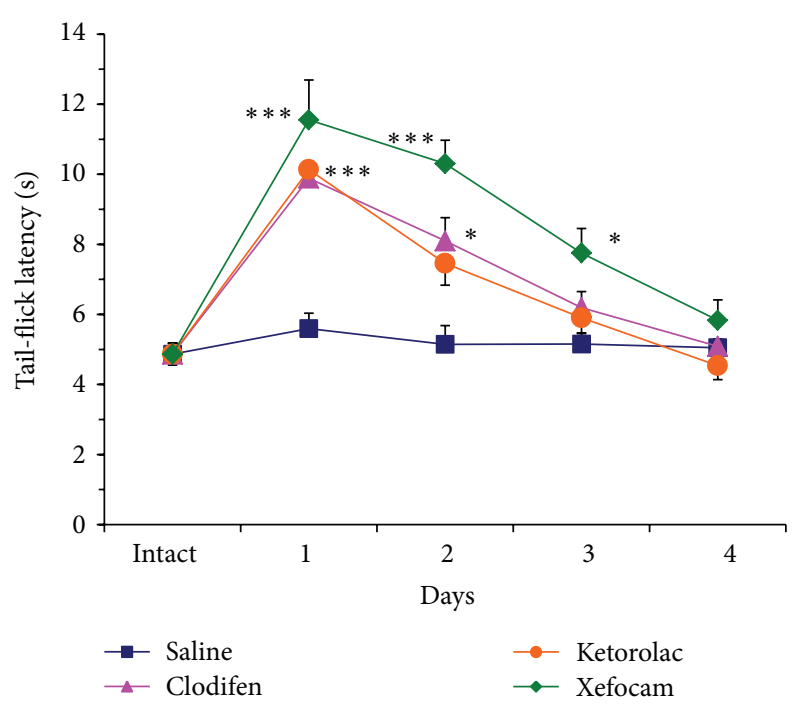

(a)

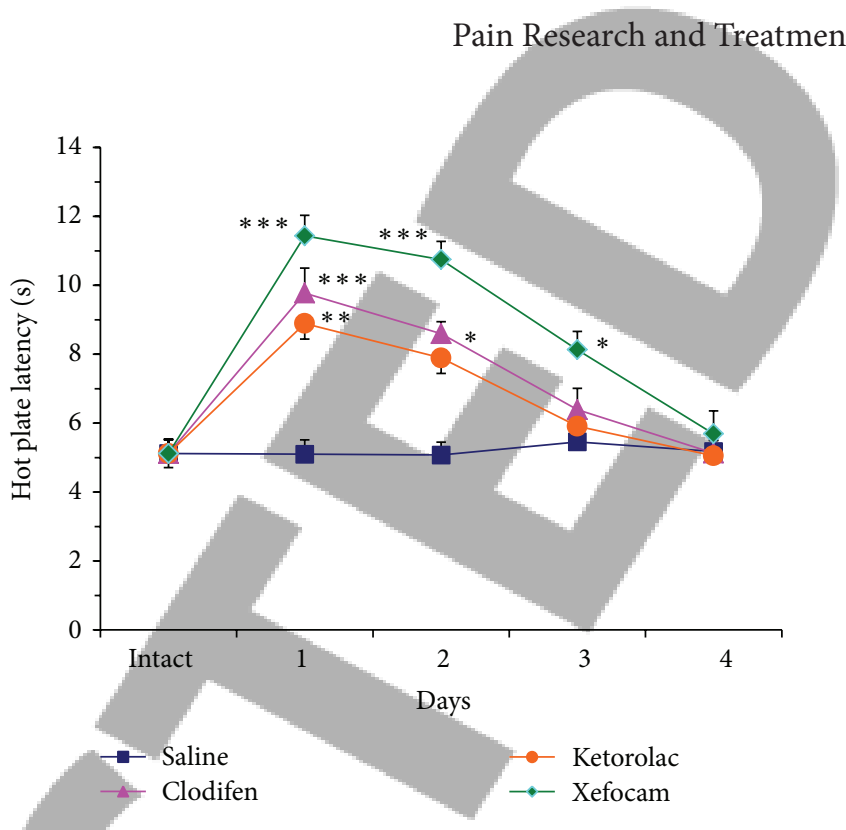

(b)

FIGURE 2: Microinjections of NSAIDs into the DH for four consecutive days result in a progressive decrease in TF (a) and HP (b) latencies as compared to vehicle saline control. The number of rats in the control group is $N=16$, in the treated groups for Clodifen $N=5$, for Ketorolac $N=6$, and for Xefocam $N=5$, respectively. ${ }^{*} P<0.05,{ }^{* *} P<0.01$, and ${ }^{* * *} P<0.001$.

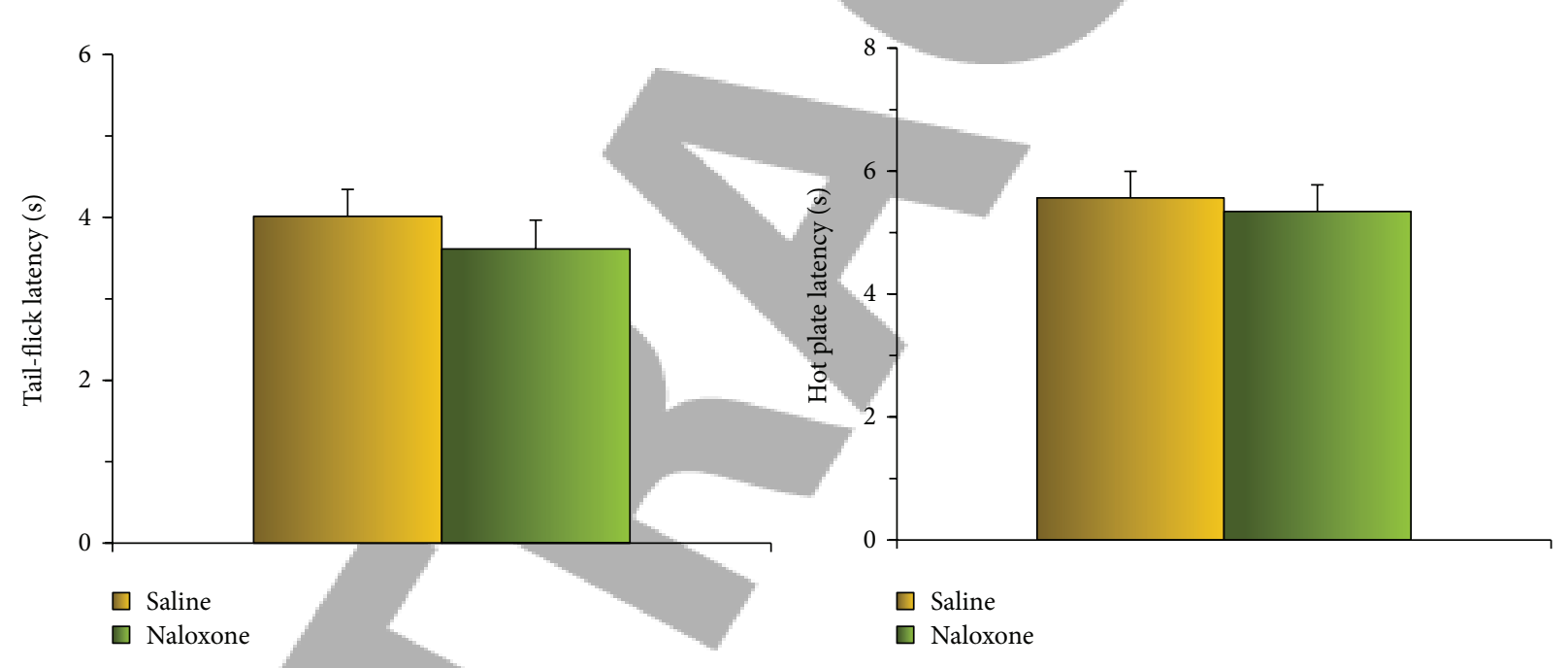

(a)

(b)

FIGURE 3: Control experiments of posttreatment with naloxone after microinjection of saline into the DH do not significantly change TF (a) and HP (b) latencies $(P>0.05)$. Number of rats is $N=5$ /group.

cross-tolerance to morphine as if opioid analgesics had been repeatedly administered [21, 22].

The mechanism producing tolerance to NSAIDs can be due to the participation of endogenous opioids and endocannabinoids $[13,24,31]$. Herein, we clearly showed that a nonselective opioid receptor antagonist naloxone significantly diminishes NSAIDs-induced antinociception. Our findings affirm the results of other investigators that microinjection of dipyrone and aspirin and systemic dipyrone are abolished by systemic injection and/or microinjections of the opioid antagonists, naloxone, and CTOP (D-phe-Cys-Tyr-Dtrp-Orn-thr-Pen-thr-NH2) $[20,28,30,32]$. The latter is a cyclic analog of the neuropeptide somatostatin and is known to block the analgesic effect of morphine [20]. Moreover, endogenous opioids are involved in the potentiation of analgesia observed with the combination of morphine plus dipyrone (metamizole). The release of endogenous opioids by dipyrone could enhance exogenous opiate effects, explaining the need for more naloxone to counteract the antinociception produced by morphine plus dipyrone [31].

The mechanisms whereby NSAIDs, in general, engage endogenous opioids are not completely understood. There is a proposed model for the PAG where $\gamma$-amino-butyric acid (GABA) containing synapses acts as the plausible site where 


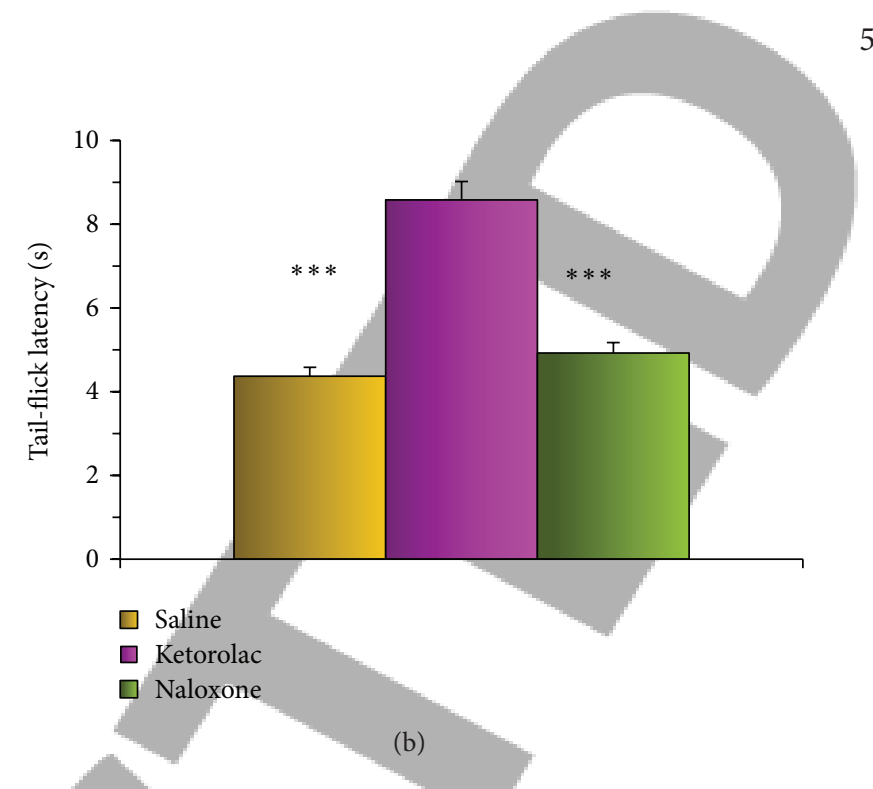

(a)
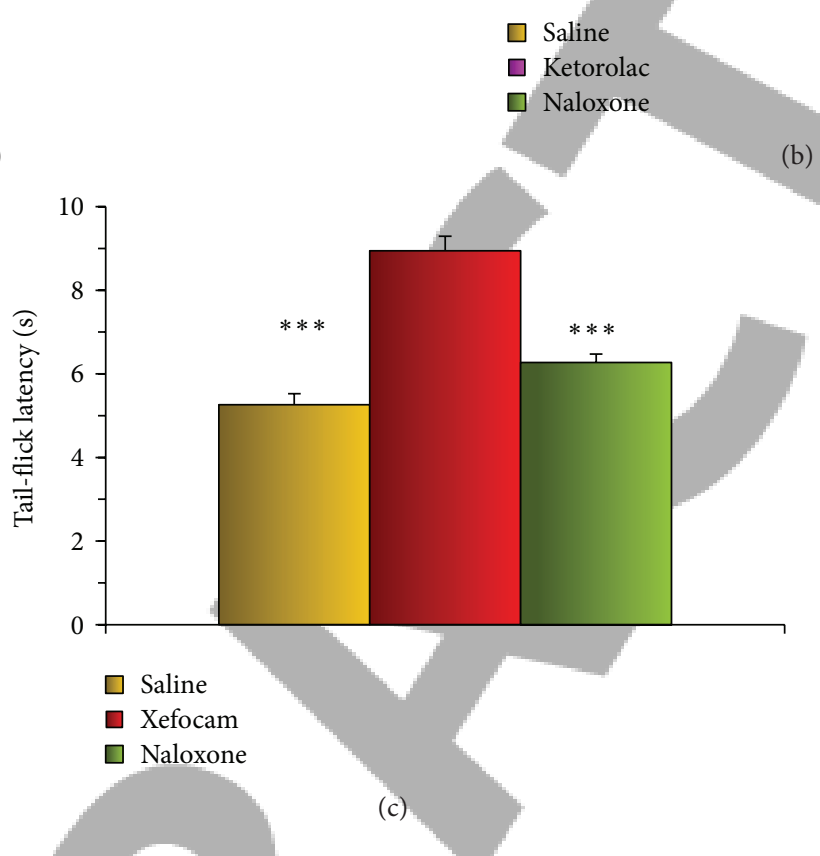

FIGURE 4: Posttreatment with naloxone after microinjections of NSAIDs into the DH results in a significant decrease in TF latency for Clodifen (a), Ketorolac (b), and Xefocam (c), respectively $(P<0.001)$. Number of rats is $N=5$ /groups. ${ }^{*} P<0.05,{ }^{* *} P<0.01$, and ${ }^{* * *} P<0.001$.

NSAIDs could converge with endogenous opioids. The PAG output neurons that drive antinociception via downstream relays, like the RVM, are tonically inhibited by local GABAergic synapses $[13,24]$. Endogenous opioids reduce presynaptic release of GABA in the PAG. Furthermore, activation of $\mu$ opioid receptors in the PAG brings about an elevation of the intracellular concentration of arachidonic acid metabolites. One of the pathways leads to the formation of hepoxilins, which increase potassium conductance. This in turn hyperpolarizes the presynaptic GABAergic terminals and decreases GABA release [32]. Disinhibition of PAG output neurons would thus drive antinociception in the downstream loop of the PAG-RVM-spinal dorsal horn [14, 33, 34]. For this pathway to function, however, an activation of $\mu$-opioid receptors seems to be necessary, because naloxone or CTOP blocks the effect of PAG-microinjected metamizole or aspirin [20, 28].

As stated above, the action of either opioid or nonopioid analgesics in the PAG leads to an excitation of PAG output neurons and this causes an activation of RVM off-cells and an inhibition of RVM on-cells, thus leading to antinociception (analgesia). When tolerance develops, PAG microinjections of morphine [35], or dipyrone [20], are no longer capable of affecting RVM neurons and inducing analgesia. These results show further neuronal relationships between opioid and nonopioid analgesics as regards the descending pain-control and modulation system [24,33]. In addition, metamizole probably can act via endocannabinoids in the downstream PAG-RVM axis reducing inflammation pain in rats [36].

There is evidence that GABAergic mediation of opioid effects is a widespread phenomenon and occurs throughout most of CNS. A colocalization between hippocampal $\mu$-opioid receptors and GABAergic interneurons in CA1, CA3, and dentate gyrus has been shown in rats [37]. The localization of $\mu$-opioid receptors on GABAergic neurons suggests that these receptors, when activated, can directly control the hippocampal GABAergic neurons' activity [37, 38]. Several studies have shown that activation of the opioid receptors can lead to the inhibition of interneuron activity resulting in diminished GABA release and the disinhibition of hippocampal pyramidal neurons [39-41].

Our results support the hypothesis that modulation of nociceptive response in the $\mathrm{DH}$ could occur in a manner 


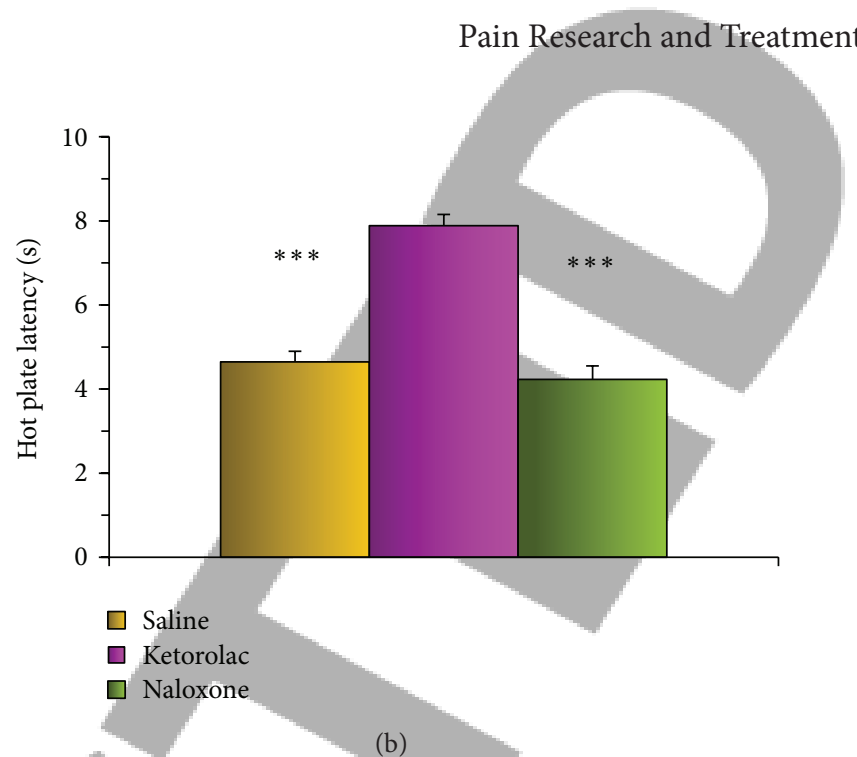

(a)

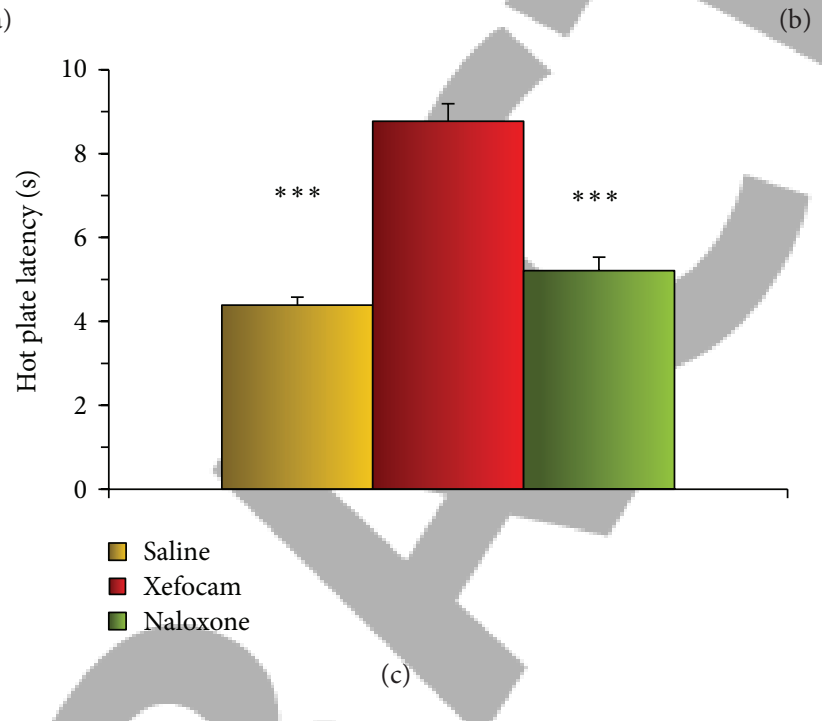

FIGURE 5: Posttreatment with naloxone after microinjections of NSAIDs into the DH results in a significant decrease in HP latency for Clodifen (a), Ketorolac (b), and Xefocam (c), respectively $(P<0.001)$. Number of rats is $N=5$ /groups. ${ }^{*} P<0.05,{ }^{* *} P<0.01$, and ${ }^{* * *} P<0.001$.

similar to that proposed for the PAG $[12-14,24,42]$. It is therefore likely that in this study the antinociception observed after microinjection of NSAIDs into the DH occurs through the inhibition of tonically active GABAergic interneurons. In addition, involvement of the downstream PAG-RVM axis mechanism is also possible.

\section{Conclusions}

In conclusion, our data showed for the first time that repeated microinjections of NSAIDs into the DH induce antinociceptive tolerance that is opioid mediated. These findings confirmed previous studies indicating that the antinociceptive action of NSAIDs may be mediated via the endogenous opioid system, as it is blocked by naloxone and exhibits tolerance.

\section{Conflict of Interests}

The authors declare that there is no conflict of interests regarding the publication of this paper.

\section{Acknowledgment}

The authors thank Dr. Marina Nebieridze for her kind assistance in histology control.

\section{References}

[1] K. D. Craig, "Emotions and psychobiology", in Wall and Mellzack's Textbook of Pain, S. B. McMahon and M. Koltzenburg, Eds., pp. 231-240, Elsevier, London, UK, 5th edition, 2006.

[2] K. Keay and R. Bandler, "Emotional and behavioral significance of the pain signal and the role of the midbrain periaqueductal gray (PAG)," in Science of Pain, A. I. Basbaum and M. C. Bushnell, Eds., pp. 627-634, Elsevier, San Diego, Calif, USA, 2009.

[3] R. A. R. Tasker, M. Choiniere, S. M. Libman, and R. Melzack, "Analgesia produced by injection of lidocaine into the lateral hypothalamus," Pain, vol. 31, no. 2, pp. 237-248, 1987.

[4] A. L. Vaccarino and R. Melzack, "Analgesia produced by injection of lidocaine into the anterior cingulum bundle of the rat," Pain, vol. 39, no. 2, pp. 237-248, 1989. 
[5] J. E. McKenna and R. Melzack, "Analgesia produced by lidocaine microinjection into the dentate gyrus," Pain, vol. 49, no. 1, pp. 105-112, 1992.

[6] L. A. Favaroni Mendes and L. Menescal-de-Oliveira, "Role of cholinergic, opioidergic and GABAergic neurotransmission of the dorsal hippocampus in the modulation of nociception in guinea pigs," Life Sciences, vol. 83, no. 19-20, pp. 644-650, 2008.

[7] M.-G. Liu and J. Chen, "Roles of the hippocampal formation in pain information processing," Neuroscience Bulletin, vol. 25, no. 5, pp. 237-266, 2009.

[8] A. A. Mutso, D. Radzicki, M. N. Baliki et al., "Abnormalities in hippocampal functioning with persistent pain," Journal of Neuroscience, vol. 32, no. 17, pp. 5747-5756, 2012.

[9] A. Erfanparast, E. Tamaddonfard, A. A. Farshid, and E. Khalilzadeh, "Antinociceptive effect of morphine microinjections into the dorsal hippocampus in the formalin-induced orofacial pain in rats," Veterinary Research Forum, vol. 1, pp. 8389, 2010.

[10] C. T. Drake, T. A. Patterson, M. L. Simmons, C. Chavkin, and T. A. Milner, "Kappa opioid receptor-like immunoreactivity in guinea pig brain: ultrastructural localization in presynaptic terminals in hippocampal formation," The Journal of Comparative Neurology, vol. 370, pp. 377-395, 1996.

[11] S. McLean, R. B. Rothman, and A. E. Jacobson, "Distribution of opiate receptor subtypes and enkephalin and dynorphin immunoreactivity in the hippocampus of squirrel, guinea pig, rat, and hamster," The Journal of Comparative Neurology, vol. 255, no. 4, pp. 497-510, 1987.

[12] H. L. Fields, A. I. Basbaum, and M. M. Heinricher, "Central nervous system mechanisms of pain modulation," in Wall and Mellzack's Textbook of Pain, S. B. McMahon and M. Koltzenburg, Eds., pp. 125-143, Elsevier, London, UK, 5th edition, 2006.

[13] M. M. Heinricher and S. L. Ingram, "The brainstem and nociceptive modulation," in Science of Pain, A. I. Basbaum and M. C. Bushnell, Eds., pp. 593-626, Elsevier, San Diego, Calif, USA, 2009.

[14] K. Ren and R. Dubner, "Descending control mechanisms," in Science of Pain, A. I. Basbaum and M. C. Bushnell, Eds., pp. 593626, Elsevier, San Diego, Calif, USA, 2009.

[15] M. G. Tsagareli, N. D. Tsiklauri, G. P. Gurtskaia, I. R. Nozadze, R. A. Kandelaki, and E. V. Abzianidze, "Tolerance effects induced by NSAID microinjections into the central nucleus of the amygdala in rats," Neurophysiology, vol. 41, no. 6, pp. 404-408, 2009.

[16] M. G. Tsagareli, N. Tsiklauri, G. Gurtskaia, I. Nozadze, and E. Abzianidze, "The central nucleus of amygdala is involved in tolerance to the antinociceptive effect of NSAIDs," Health, vol. 2, pp. 64-68, 2010.

[17] M. G. Tsagareli, I. Nozadze, N. Tsiklauri, and G. Gurtskaia, "Tolerance to non-opioid analgesics is opioid sensitive in the nucleus raphe magnus," Frontiers in Neuroscience, vol. 5, article 92, 2011.

[18] N. Tsiklauri, I. Nozadze, G. Gurtskaia, V. Berishvili, E. Abzianidze, and M. Tsagareli, "Tolerance induced by non-opioid analgesic microinjections into rat's periaqueductal gray and nucleus raphe," Georgian Medical News, no. 180, pp. 47-55, 2010.

[19] N. D. Tsiklauri, I. R. Nozadze, G. P. Gurtskaia, and M. G. Tsagareli, "Opioid sensitivity of analgesia induced by microinjections of nonsteroidal anti-inflammatory drugs into the nucleus raphe magnus," Neurophysiology, vol. 43, no. 3, pp. 213216, 2011.
[20] V. Tortorici, Y. Aponte, H. Acevedo, L. Nogueira, and H. Vanegas, "Tolerance to non-opioid analgesics in PAG involves unresponsiveness of medullary pain-modulating neurons in male rats," European Journal of Neuroscience, vol. 29, no. 6, pp. 1188-1196, 2009.

[21] V. Tortorici and H. Vanegas, "Opioid tolerance induced by metamizol (dipyrone) microinjections into the periaqueductal grey of rats," European Journal of Neuroscience, vol. 12, no. 11, pp. 4074-4080, 2000.

[22] M. G. Tsagareli and N. Tsiklauri, Behavioral Study of "NonOpioid Tolerance", Nova Biomedical Publishers, New York, NY, USA, 2012.

[23] M. G. Tsagareli, N. Tsiklauri, I. Nozadze, and G. Gurtskaia, "Tolerance effects of NSAIDs microinjected into central amygdala, periaqueductal grey, and nucleus raphe: possible cellular mechanism," Neural Regeneration Research, vol. 7, pp. 10291039, 2012.

[24] H. Vanegas, E. Vazquez, and V. Tortorici, "NSAIDS, opioids, cannabinoids and the control of pain by the central nervous system," Pharmaceuticals, vol. 3, no. 5, pp. 1335-1347, 2010.

[25] V. Duric and K. E. McCarson, "Effects of analgesic or antidepressant drugs on pain- or stress-evoked hippocampal and spinal neurokinin-1 receptor and brain-derived neurotrophic factor gene expression in the rat," Journal of Pharmacology and Experimental Therapeutics, vol. 319, no. 3, pp. 1235-1243, 2006.

[26] M. Zimmermann, "Ethical guidelines for investigations of experimental pain in conscious animals," Pain, vol. 16, no. 2, pp. 109-110, 1983.

[27] G. Paxinos and C. Watson, The Rat Brain in Stereotaxic Coordinates, Academic Press, San Diego, Calif, USA, 1997.

[28] A. J. Pernia-Andrade, V. Tortorici, and H. Vanegas, "Induction of opioid tolerance by lysine-acetylsalicylate in rats," Pain, vol. 111, no. 1-2, pp. 191-200, 2004.

[29] N. Tsiklauri, V. Viatchenko-Karpinski, N. Voitenko, and M. G. Tsagareli, "Non-opioid tolerance in juvenile and adult rats," European Journal of Pharmacology, vol. 629, no. 1-3, pp. 68-72, 2010.

[30] E. Vazquez, N. Hernandez, W. Escobar, and H. Vanegas, "Antinociception induced by intravenous dipyrone (metamizol) upon dorsal horn neurons: involvement of endogenous opioids at the periaqueductal gray matter, the nucleus raphe magnus, and the spinal cord in rats," Brain Research, vol. 1048, no. 1-2, pp. 211-217, 2005.

[31] G. P. Hernández-Delgadillo and S. L. Cruz, "Endogenous opioids are involved in morphine and dipyrone analgesic potentiation in the tail flick test in rats," European Journal of Pharmacology, vol. 546, no. 1-3, pp. 54-59, 2006.

[32] C. W. Vaughan, "Enhancement of opioid inhibition of GABAergic synaptic transmission by cyclo-oxygenase inhibitors in rat periaqueductal grey neurones," British Journal of Pharmacology, vol. 123, no. 8, pp. 1479-1481, 1998.

[33] M. W. Wessendorf, C. W. Vaughan, and H. Vanegas, "Rethinking the PAG and RVM: supraspinal modulation of nociception by opioids and nonopioids," in Proceedings of the 11th World Congress Pain, H. Flor, E. Kalso, and J. O. Dostrovsky, Eds., pp. 311-320, IASP Press, Seattle, Wash, USA, 2006.

[34] M. M. Morgan, K. L. Whittier, D. M. Hegarty, and S. A. Aicher, "Periaqueductal gray neurons project to spinally projecting GABAergic neurons in the rostral ventromedial medulla," Pain, vol. 140, no. 2, pp. 376-386, 2008.

[35] V. Tortorici, M. M. Morgan, and H. Vanegas, “Tolerance to repeated microinjection of morphine into the periaqueductal 
gray is associated with changes in the behavior of off- and oncells in the rostral ventromedial medulla of rats," Pain, vol. 89, no. 2-3, pp. 237-244, 2001.

[36] W. Escobar, K. Ramirez, C. Avila, R. Limongi, H. Vanegas, and E. Vazquez, "Metamizol, a non-opioid analgesic, acts via endocannabinoids in the PAG-RVM axis during inflammation in rats," European Journal of Pain, vol. 16, pp. 676-689, 2012.

[37] A. E. Kalyuzhny and M. W. Wessendorf, "Relationship of muand delta-opioid receptors to GABAergic neurons in the central nervous system, including antinociceptive brainstem circuits," The Journal of Comparative Neurology, vol. 392, pp. 528-547, 1998.

[38] A. E. Kalyuzhny and M. W. Wessendorf, "CNS GABA neurons express the $\mu$-opioid receptor: immunocytochemical studies," NeuroReport, vol. 8, no. 15, pp. 3367-3372, 1997.

[39] K. R. Svoboda and C. R. Lupica, "Opioid inhibition of hippocampal interneurons via modulation of potassium and hyperpolarization-activated cation $(\mathrm{I}(\mathrm{h})$ ) currents," Journal of Neuroscience, vol. 18, no. 18, pp. 7084-7098, 1998.

[40] G. A. Cohen, V. A. Doze, and D. V. Madison, "Opioid inhibition of GABA release from presynaptic terminals of rat hippocampal interneurons," Neuron, vol. 9, no. 2, pp. 325-335, 1992.

[41] C. R. Lupica, W. R. Proctor, and T. V. Dunwiddie, "Dissociation of $\mu$ and $\delta$ opioid receptor-mediated reductions in evoked and spontaneous synaptic inhibition in the rat hippocampus in vitro," Brain Research, vol. 593, no. 2, pp. 226-238, 1992.

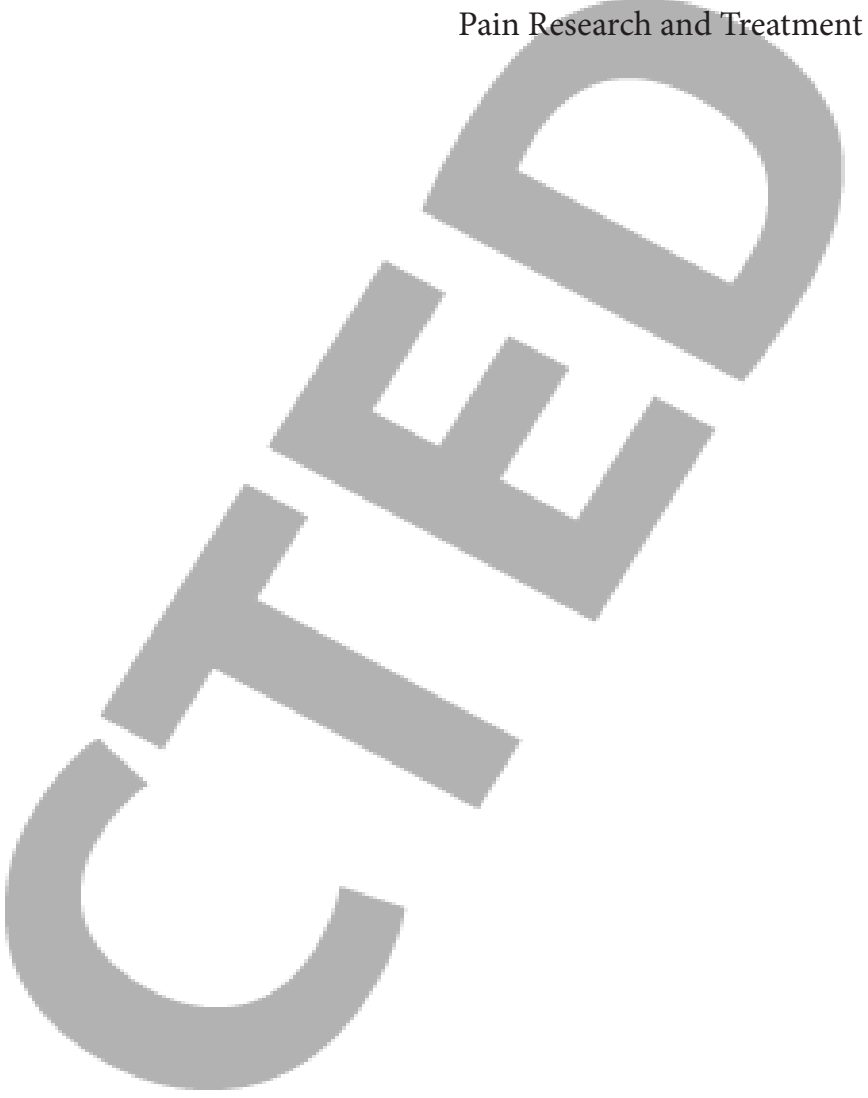

[42] M. M. Heinricher, I. Tavares, J. L. Leith, and B. M. Lumb, "Descending control of nociception: specificity, recruitment and plasticity," Brain Research Reviews, vol. 60, no. 1, pp. 214225, 2009. 